\title{
Subgap Two-Photon States in Polycyclic Aromatic Hydrocarbons: Evidence for Strong Electron Correlations
}

\author{
Karan Aryanpour, ${ }^{1, \text { f }}$ Adam Roberts, ${ }^{2,3}$ Arvinder Sandhu, ${ }^{1,2}$ \\ Rajendra Rathore, ${ }^{4}$ Alok Shukla, ${ }^{5}$ and Sumit Mazumdar*1,2 \\ ${ }^{1}$ Department of Physics, University of Arizona, Tucson, Arizona 85721, United States \\ ${ }^{2}$ College of Optical Sciences, University of Arizona, Tucson, Arizona 85721, United States \\ ${ }^{3}$ U.S. Army Aviation and Missile Research, Development, and Engineering Center, \\ Redstone Arsenal, Huntsville, Alabama 35898, United States \\ ${ }^{4}$ Department of Chemistry, Marquette University, Milwaukee, Wisconsin 53201, United States \\ ${ }^{5}$ Department of Physics, Indian Institute of Technology, Powai, Mumbai - 400076, India
}

(Dated: June 25, 2021)

\begin{abstract}
Strong electron correlation effects in the photophysics of quasi-one-dimensional $\pi$-conjugated organic systems such as polyenes, polyacetylenes, polydiacetylenes, etc., have been extensively studied. Far less is known on correlation effects in two-dimensional $\pi$-conjugated systems. Here we present theoretical and experimental evidence for moderate repulsive electron-electron interactions in a number of finite polycyclic aromatic hydrocarbon molecules with $D_{6 h}$ symmetry. We show that the excited state orderings in these molecules are reversed relative to that expected within oneelectron and mean-field theories. Our results reflect similarities as well as differences in the role and magnitude of electron correlation effects in these two-dimensional molecules compared to those in polyenes.
\end{abstract}

\section{- INTRODUCTION}

Strong electron correlation effects remain a highly relevant topic in the physics and chemistry of carbonbased materials. Extensive experimentation has confirmed strong electron correlation effects in quasi-onedimensional (quasi-1D) carbon-based systems such as linear $\pi$-conjugated polymers, single-walled carbon nanotubes, graphene nanoribbons, etc. Historically, the study of the excited state ordering in finite polyenes 145] provided the most convincing evidence for strong electron correlation effects in quasi-1D $\pi$-conjugated systems, and formed the basis of correlated-electron theories in the long chain polyacetylenes and polydi-

2D Polycyclic Aromatic Hydrocarbons (PAHs):
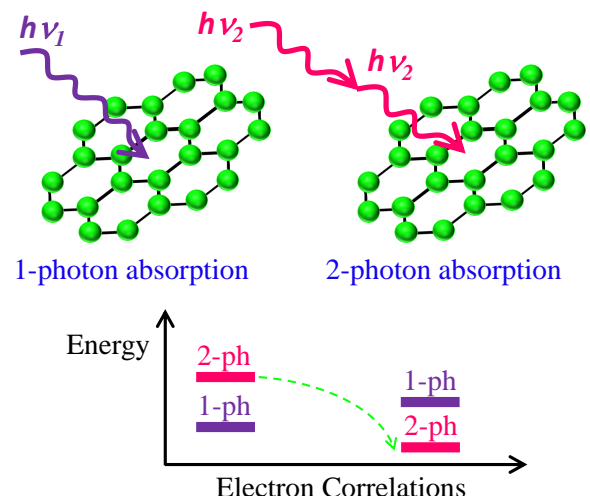

Table of Contents acetylenes. 6, 7] The early calculations [3-5] were within the semiempirical Pariser-Parr-Pople (PPP) $\pi$-electron model Hamiltonian, [8, 9] within which eigenstates of even polyenes with $\mathrm{C}_{2 h}$ point group symmetry have even and odd spatial as well as charge-conjugation symmetries (CCSs), and allowed one-photon (two-photon) transitions from the $1^{1} \mathrm{~A}_{g}^{-}$ground state are to ${ }^{1} \mathrm{~B}_{u}^{+}\left({ }^{1} \mathrm{~A}_{g}^{-}\right)$states with opposite (same) symmetries (the superscript 1 indicates singlet spin state and the "plus" and "minus" superscripts refer to CCS). The occurrence of the lowest two-photon state, the $2^{1} \mathrm{~A}_{g}^{-}$, below the one-photon $1^{1} \mathrm{~B}_{u}^{+}$ in polyenes [1, 2] is opposite to that expected within the one-electron Hückel and mean-field Hartree-Fock (HF) theories and is a consequence of strong shortrange electron-electron $(\mathrm{e}-\mathrm{e})$ interactions within the PPP model. 3 5] Subsequent to these early model calculations, considerable effort has gone into developing more sophisticated quantum chemical correlated $a b$ initio approaches to polyene spectra. Quantum chemical approaches that are now able to reproduce the correct one-photon versus two-photon state ordering for short linear polyenes (usually up to octatetraene) include the complete active space second order perturbation theory (CASPT2) and third-order coupled cluster theory, 10, 11 and the extended algebraic diagrammatic construction (ADC(2)-x) method. [12 14] Correct excited state ordering is also found within a density functional theory-based multiple reference configuration interaction (DFT-MRCI) approach. 15] CCS characteristic of the PPP model is absent within the latter approaches; however, the violation of CCS is weak. 16 Importantly, the quantum chemical approaches do find the double excitation character of the $2^{1} \mathrm{~A}_{g}$. Theoretical works have also found reversed excited state ordering in long acenes from pentacene onward, within the PPP model, [17, 18 a semiempirical DFT-MRCI method [19] and the ADC(2)-x ap-

*Electronic address: karana@physics.arizona.edu 


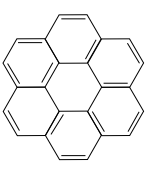

(a) Coronene

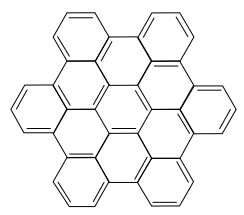

(b) $\mathrm{HBC}$

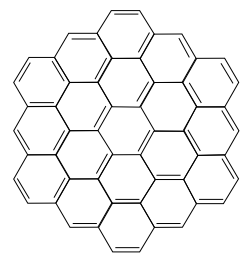

(c) Circumcoronene
FIG. 1: PAH molecules studied in this work: (a) coronene $\left(\mathrm{C}_{24} \mathrm{H}_{12}\right)$ (b) hexa-peri-hexabenzocoronene, hereafter HBC $\left(\mathrm{C}_{42} \mathrm{H}_{18}\right)$, and $(\mathrm{d})$ circumcoronene $\left(\mathrm{C}_{54} \mathrm{H}_{18}\right)$.

proach. 20] Acenes can be considered as coupled polyene chains and are also quasi-1D.

In contrast to the quasi-1D systems, there have been few, if any, systematic studies of electron correlation effects in two-dimensional (2D) molecules. In this work we take the first step toward addressing electron correlation effects in $2 \mathrm{D}$ molecules by conducting a systematic study of excited states ordering in polycyclic aromatic hydrocarbons (PAHs). By performing meticulous experimental and computational analyses of the one- and two-photon excited states for the three PAH molecules shown in Figure $1 \mathrm{a}-\mathrm{c}$, we find reversed excited state ordering within these $2 \mathrm{D}$ molecules in clear contradiction to the description of one-electron theory. These correlation effects are found to be overall weaker compared to quasi-1D carbon-based systems, due to the larger oneelectron bandwidth in 2D. Possible extension of our study to larger molecules can also shed light on electron correlation effects in graphene as a genuine 2D carbon-based system in the thermodynamic limit. Within the Hückel tight-binding $\pi$-electron theory graphene is a semimetal, with linear energy versus momentum relationship near the Dirac point where the filled valence and empty conduction bands meet. Although many experimental observations appear to be in agreement with this description, 21, 22 the neglect of the repulsive $\mathrm{e}-\mathrm{e}$ interaction between the $\pi$-electrons is increasingly being questioned. [23, 24

\section{— THEORETICAL MODEL AND METHODS}

\section{Pariser-Parr-Pople Model}

Quantum chemical approaches can correctly describe low-lying two-photon states of double excitation charac- ter only for relatively small molecules. [10, 17, 19, 20, The two larger PAH molecules of interest here, namely, HBC and circumcoronene, lie outside the scope of these calculations. Moreover, while we are principally interested in the relative energies of the lowest one- and two-photon states, we will in the following compare experimental and calculated two-photon absorption (TPA) spectra over a broad frequency range, which requires that we are able to calculate the energies and wave functions of the higher energy two-photon states. These requirements restrict us to perform our calculations within the semiempirical PPP Hamiltonian, 8, 9] which is written as

$$
\begin{aligned}
& H_{P P P}=-t \sum_{\langle i j\rangle \sigma}\left(c_{i \sigma}^{\dagger} c_{j \sigma}+c_{j \sigma}^{\dagger} c_{i \sigma}\right)+U \sum_{i} n_{i \uparrow} n_{i \downarrow}+ \\
& \sum_{i<j} V_{i j}\left(n_{i}-1\right)\left(n_{j}-1\right),
\end{aligned}
$$

where $c_{i \sigma}^{\dagger}$ creates a $\pi$-electron of spin $\sigma$ on carbon atom $i$, $n_{i \sigma}=c_{i \sigma}^{\dagger} c_{i \sigma}$ is the number of electrons of spin $\sigma$ on atom $i$, and $n_{i}=\sum_{\sigma} n_{i \sigma}$. The one-electron hopping integral $t$ is between nearest neighbor carbon atoms $i$ and $j, U$ is the Hubbard repulsion between two electrons occupying the same atomic $p_{z}$ orbital, and $V_{i j}$ is the long-range intersite Coulomb interaction. We choose standard $t=2.4$ $\mathrm{eV}$, 44 6] and obtain $V_{i j}$ from the parametrization, 25] $V_{i j}=U / \kappa \sqrt{1+0.6117 R_{i j}^{2}}$, where $R_{i j}$ is the distance in $\AA$ between carbon atoms $i$ and $j$ and $\kappa$ is an effective dielectric constant. We fix carbon-carbon $(\mathrm{C}-\mathrm{C})$ bond lengths to $1.4 \AA$ and all bond angles to $120^{\circ}$. The actual bond lengths and angles may deviate slightly from these mean values, but given the aromatic nature of the molecules, we expect these deviations and their effects on the energies of the one- and two-photon states to be small. This is shown explicitly for coronene (see Appendix), the only molecule for which the experimental bond lengths are available in the literature. We choose $U=8 \mathrm{eV}$ and $\kappa=2$ based on the excellent fits obtained previously with these parameters for the excited states of poly-paraphenylenevinylene, 25] polyacenes, 26] and single-walled carbon nanotubes. 27.

The molecules in Figure 1 belong to the $D_{6 h}$ point group and possess inversion, reflection, 6-fold rotational, and CC symmetries. Since $D_{6 h}$ is not Abelian, in the following we adopt the Abelian $D_{2 h}$ point group symmetry to partition excited states into distinct one- and twophoton classes. The ground state is in the ${ }^{1} \mathrm{~A}_{g}^{-}$subspace for all the molecules of Figure $1 \mathrm{a}-\mathrm{c}$. Dipole selection rules dictate that one-photon (linear) absorption (TPA) occur only to ${ }^{1} \mathrm{~B}_{2 u}^{+}$and ${ }^{1} \mathrm{~B}_{3 u}^{+}\left({ }^{1} \mathrm{~A}_{g}^{-}\right.$and $\left.{ }^{1} \mathrm{~B}_{1 g}^{-}\right)$states with spatial and $\mathrm{CC}$ symmetries opposite to (the same as) that of the ground state. In addition PAHs also have ${ }^{1} \mathrm{~B}_{2 u}^{-}$ and ${ }^{1} \mathrm{~B}_{3 u}^{-}$states forbidden in both one- and two-photon processes. 26, 27. 


\section{Multiple-Reference Configuration Interaction Approach}

Our calculations were done using the multiple reference singles and doubles configuration interaction (MRSDCI) 5. The MRSDCI retains the dominant single, double, triple, and quadruple excitations for each targeted state [5, with the number of configurations systematically increased until numerical convergence is reached. While excitonic effects due to the correlation between a single electron-hole pair in optically allowed states have been treated very successfully for graphene 28 and other carbon nanostructures 29 31 within the GW-Bethe Salpeter technique, application of this first-principles approach to investigate the lowest two-photon eigenstates has not yet been possible. For example, the lowest twophoton states that occur below the optical gap in transpolyacetylene 6 is missed within the GW-Bethe Salpeter approach, 32] even though the latter gives accurate description of the optical exciton. Thus, the inclusion of CI with multiple electron-hole excitations is a necessary requirement for obtaining two-photon states below the lowest one-photon optical state. [1, 3, 6, 10,17, 19, 20] The semiempirical approach taken by us cannot be easily extended to the thermodynamic limit (except in one dimension, where the density matrix renormalization group approach can be used [7, 33, 34]), but this disadvantage is offset by the ability to include high order correlation effects, involving triply and quadruply excited configurations with respect to the restricted Hartree-Fock (RHF) reference state. Next, we describe the procedure involved in performing various levels of CI calculations presented in this work.

The initial step of our CI studies involves determination of the self-consistent RHF solutions of the PPP Hamiltonian (see eq1). The basis functions of the manybody calculations are configurations with all possible electron occupancies of the HF molecular orbitals (MOs). A full CI calculation involves construction of the Hamiltonian matrix with all possible excitations from the HF ground state, and this is the procedure we have followed in the text for octatetraene and decapentaene. For select few cases, quadruple-CI (QCI), which incorporates all excitations up to quadruples from the HF ground state, is also possible. With a large number of electrons, as in the present case, one then encounters the following problem. On one hand, straightforward high-order CI such as the QCI is beyond today's computational capability. On the other hand, lower order CI such as double-CI does not give the correct excited state ordering for large molecules. It is precisely for such large systems that the MRSDCI is used. [35, 36] We have previously performed calculations as large as the ones reported here for quasi-1D molecules and oligomers. $37+43$.

The MRSDCI is performed in two steps. At the first step, a few $\left(N_{r e f}\right)$ singly and doubly excited reference configurations that best describe the excited states in the targeted symmetry subspace are selected on the basis of a trial double-CI calculation. The second step involves the MRSDCI calculation, in which the Hamiltonian matrix consists of single and double excitations with respect to the original $N_{r e f}$ configurations themselves. The total number of configurations $N_{\text {total }}$ therefore includes also the subset of the dominant triple and quadruple excitations. The new set of $N_{\text {total }}$ configurations are now examined, single and double excitations from the HF ground states that contribute significantly to the eigenstates within the targeted symmetry subspace are identified, and these are included to augment the new reference space, following which the MRSDCI is performed again. This iterative approach is continued, with updating of $N_{\text {ref }}$ and $N_{\text {total }}$ at every step, until convergence to some tolerance determined by the size of the system is reached.

In Table I we have given the final $N_{\text {ref }}$ and $N_{\text {total }}$ reached in our MRSDCI calculations for all four $D_{2 h}$ subspaces relevant for the calculations of nonlinear absorptions in coronene, HBC and circumcoronene (for the ${ }^{1} \mathrm{~A}_{g}$ subspace of coronene the calculation was done using QCI). To the best of our knowledge, these are the largest CI calculations performed to date for the excited states of these molecules.

\section{Calculation of the Two-Photon Absorption Spectrum}

The TPA is calculated from the imaginary component of the third-order nonlinear optical susceptibility $\chi^{(3)}(\omega, \omega, \omega,-\omega)$, the resonant contribution $\chi^{(3)}(T P A)$ of which is given by the sum-overstate expression, 44 .

$$
\begin{array}{r}
\chi_{i j k l}^{(3)}(T P A)=\frac{N}{6 \hbar^{3}}\left(\sum _ { m n p } ^ { \prime } \left[\mu_{g n}^{i} \mu_{n m}^{l} \mu_{m p}^{k} \mu_{p g}^{j}+\right.\right. \\
\left.\mu_{g n}^{i} \mu_{n m}^{l} \mu_{m p}^{j} \mu_{p g}^{k}+\mu_{g n}^{l} \mu_{n m}^{i} \mu_{m p}^{k} \mu_{p g}^{j}+\mu_{g n}^{l} \mu_{n m}^{i} \mu_{m p}^{j} \mu_{p g}^{k}\right] \times \\
\frac{1}{\left(\omega_{n g}-\omega\right)\left(\omega_{m g}-2 \omega-i \delta\right)\left(\omega_{p g}-\omega\right)}-\sum_{n p}\left[\mu_{g n}^{i} \mu_{n g}^{j} \mu_{g p}^{l} \mu_{p g}^{k}\right. \\
\left.+\mu_{g n}^{i} \mu_{n g}^{k} \mu_{g p}^{l} \mu_{p g}^{j}+\mu_{g n}^{l} \mu_{n g}^{k} \mu_{g p}^{i} \mu_{p g}^{j}+\mu_{g n}^{l} \mu_{n g}^{j} \mu_{g p}^{i} \mu_{p g}^{k}\right] \times \\
\left.\frac{1}{\left(\omega_{n g}-\omega\right)\left(\omega_{p g}-\omega\right)\left(\omega_{p g}-\omega\right)}\right),
\end{array}
$$

where $N$ is the number of molecules, $g$ is the ground state, $n$ and $p$ are virtual one-photon states, and $m$ is twophoton states. $\delta$ is the line width of the spectrum when $\omega \approx \omega_{m g} / 2$ and was set to $0.03 \mathrm{eV}$ in all our $\chi^{(3)}(T P A)$ calculations for various PAH molecules in this work. Here $\mu_{g n}^{i}$ is the $i$ th component of the matrix element of the transition dipole operator between states $g$ and $n$. Other components of the transition dipole matrix elements are defined similarly, and the prime over the first summation indicates $m \neq g$. With $D_{2 h}$ symmetry, TPAs are to ${ }^{1} \mathrm{~A}_{g}^{-}$and ${ }^{1} \mathrm{~B}_{1 g}^{-}$states, which are nondegenerate when the Coulomb interactions are nonzero. The matrix element 


\begin{tabular}{l|rrrrrr} 
symmetry & \multicolumn{2}{|c}{ coronene } & \multicolumn{2}{c}{ HBC } & \multicolumn{2}{c}{ circumcoronene } \\
& $N_{\text {ref }}$ & $N_{\text {total }}$ & $N_{\text {ref }}$ & $N_{\text {total }}$ & $N_{\text {ref }}$ & $N_{\text {total }}$ \\
\hline & & & & & & \\
\\
${ }^{1} \mathrm{~B}_{2 u}:$ & 215 & 1082466 & 102 & 2227463 & 77 & 3645309 \\
${ }^{1} \mathrm{~B}_{3 u}:$ & 202 & 972754 & 88 & 1719854 & 70 & 3133234 \\
${ }^{1} \mathrm{~A}_{g}:$ & $1^{b}$ & 2045687 & 184 & 3371103 & 100 & 3864837 \\
${ }^{1} \mathrm{~B}_{1 g}:$ & 268 & 1162244 & 168 & 3167504 & 148 & 3745386
\end{tabular}

TABLE I: Number of Final Reference Configurations, $N_{r e f}$, and the Dimension of the Hamiltonian Matrix, $N_{t o t a l}$, for the PAH Molecules of Figure $1 \beta-c$ for the $D_{2 h}$ Symmetry Subspaces Relevant to Linear and Nonlinear Optics ${ }^{a}$

${ }^{a}$ The final MRSDCI wavefunctions contained basis functions with coefficients as small as 0.04, 0.04 and 0.06 for coronene, HBC and circumcoronene, respectively. ${ }^{b} \mathrm{QCI}$

of the $x$-component of the transition dipole operator is nonzero only between the ${ }^{1} \mathrm{~A}_{g}^{-}$and the ${ }^{1} \mathrm{~B}_{3 u}^{+}$states. As a consequence, two-photon resonances in the 1111, 2222, 1221, and 2112 components of $\chi^{(3)}(T P A)$ in eq 2 are all to ${ }^{1} \mathrm{~A}_{g}^{-}$states only. Similarly, the matrix element of the $y$-component of the transition dipole operator is nonzero between ${ }^{1} \mathrm{~B}_{3 u}^{+}$and ${ }^{1} \mathrm{~B}_{1 g}^{-}$states only. Hence, two-photon resonances in the $1212,2121,1122$, and 2211 components of $\chi^{(3)}(T P A)$ are all to ${ }^{1} \mathrm{~B}_{1 g}^{-}$states. In eq 2 above, the intermediate one-photon virtual state of ${ }^{1} \mathrm{~B}_{1 u}^{+}$and two-photon states of ${ }^{1} \mathrm{~B}_{2 g}^{-}$, and ${ }^{1} \mathrm{~B}_{3 g}^{-}$do not contribute because they all involve matrix elements of the $z$ component (out of plane) of the dipole operator; due to the planar structure of PAH molecules studied in this work, these dipole transition matrix elements are vanishingly small. The experimental solution-phase PA spectrum is compared against the orientational average of the gasphase TPA susceptibilities [45],

$$
\begin{aligned}
\chi_{\text {avg }}^{(3)}(T P A)=\frac{1}{5} & {\left[\sum_{i} \chi_{i i i i}^{(3)}(T P A)+\frac{1}{3}\left(\sum _ { i \neq j } \left(\chi_{i i j j}^{(3)}(T P A)\right.\right.\right.} \\
& \left.\left.+\chi_{i j i j}^{(3)}(T P A)+\chi_{i j j i}^{(3)}(T P A)\right)\right] .
\end{aligned}
$$

\section{RESULTS: THEORY VERSUS EXPERIMENT}

Hückel Calculations. Before presenting the results of the correlated-electron calculations, we discuss the results of calculations based on the Hückel model which is the $U=V_{i j}=0$ limit of the PPP model. The goal here is to demonstrate that the Hückel model fails qualitatively to give the excited state ordering that is observed experimentally (see below). In Figure $2 a-c$ we show the frontier MOs for coronene, HBC and circumcoronene, respectively, where we have indicated the highest occupied and lowest unoccupied MOs (HOMO and LUMO) and the lowest one and two-photon allowed one electron-one hole $(1 \mathrm{e}-1 \mathrm{~h})$ transitions $(1 \mathrm{e}-1 \mathrm{~h}$ two-photon transitions that originate from MOs below the HOMO and are related to the ones shown in the figure by CCS are not shown). Note that all energies are in units of $|t|$ and dimensionless here. Two electron-two hole $(2 \mathrm{e}-2 \mathrm{~h})$ twophoton states lie outside the region of experimental interest and are hence not shown. Optically bright and dark states of $\mathrm{B}_{u}$ symmetry are degenerate in the Hückel limit. The two-photon ${ }^{1} \mathrm{~A}_{g}^{-}$and ${ }^{1} \mathrm{~B}_{1 g}^{-}$states are also degenerate.

The hopping integral $t$ determines all orbital energies and energy gaps within the Hückel model. It is thus possible to fit the allowed optical gap for each molecule by choosing an appropriate $t$, although this may need slightly different $t$ values for different molecules. Furthermore, in the absence of correlation contribution to the optical gap, it is only natural that the absolute value of $|t|$ needed to fit the optical gap within the Hückel model will be larger than in the PPP model. Fitting the peak energy $4.1 \mathrm{eV}$ of the strong optical absorption in coronene within the Hückel theory requires $t=3.8$ $\mathrm{eV}$. The calculated linear absorption spectrum with the larger $|t|$ is shown in Figure 2d. Figure $2 \mathrm{~d}$ shows our plot of $\operatorname{Im}\left[\chi_{\text {avg }}^{(3)}(T P A)\right]$ (see eq 3 for coronene, which was evaluated using the exact Hückel wave functions and the sum-overstates procedure of eq 2. The lowest twophoton state in Figure $2 \mathrm{~d}$ is $1.75 \mathrm{eV}$ above the optical one-photon state in coronene. In discussions of excited state ordering, the focus is usually on energetics alone. We emphasize that the density of two-photon states and the intensities of the TPAs also reveal correlation effects, 46. 47] and note that in Figure $2 \mathrm{~d}$ the TPA is most intense at the lowest energy.

Figure 2e shows the Hückel plots of linear absorption and TPA in HBC, again with the calculated one-photon gap matching the experimental gap. The required $t$ is now $3.6 \mathrm{eV}$, and the lowest two-photon state is $0.55 \mathrm{eV}$ above the one-photon state. We chose the same $t=3.8$ $\mathrm{eV}$ in our calculations for circumcoronene as in coronene (Hückel calculation of circumcoronene with this $t$ reproduces the optical gap obtained with PPP-MRSDCI and $t=2.4 \mathrm{eV}$, as given in Table II below). The calculated Hückel linear and TPA spectra for circumcoronene are shown in Figure 2f. Once again, the lowest two-photon state is considerably higher than the one-photon state, and as in coronene the higher energy two-photon state has a weaker intensity than the lower one. The similari- 


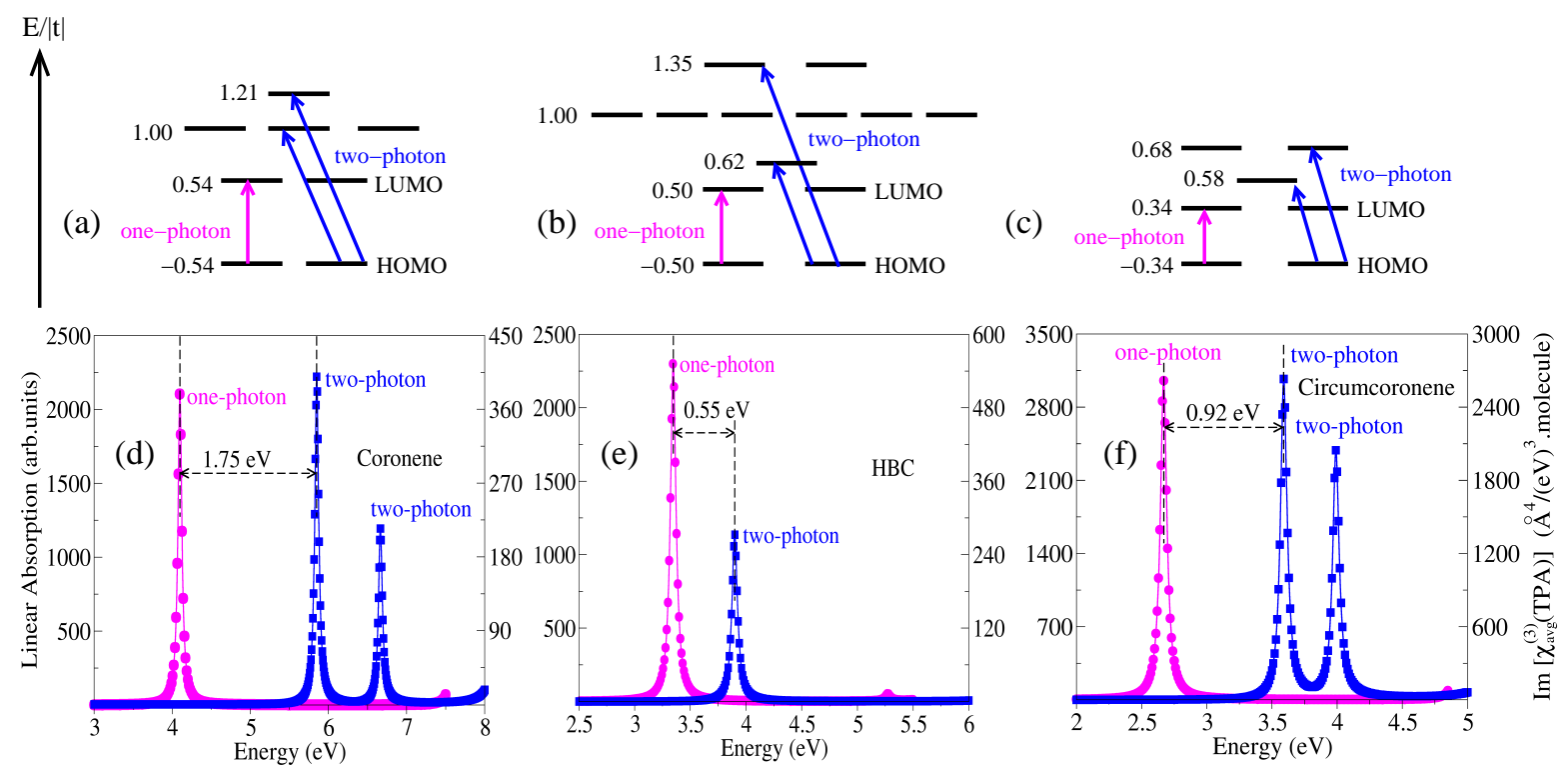

FIG. 2: Frontier MOs of (a) coronene, (b) HBC, and (c) circumcoronene, with the lowest one-photon and two-photon allowed $1 \mathrm{e}-1 \mathrm{~h}$ excitations indicated by arrows in the noninteracting Hückel limit. Panels (d) - (f) present the calculated linear and TPA spectra corresponding to molecules of panels (a)-(c), respectively. Two-photon transitions shown are at twice the fundamental frequencies, for comparison to the one-photon transitions. The $t_{i j}$ used in the calculations are $3.8 \mathrm{eV}$ for both coronene and circumcoronene and $3.6 \mathrm{eV}$ for HBC. These values were arrived at by fitting the peak energies of the strong optical absorptions in the experimental linear spectra of coronene and HBC, and the calculated many-body optical gap in circumcoronene against the Hückel theory (see text). $\delta$ in eq 2 is $0.03 \mathrm{eV}$ for all the TPA spectra of panels (d)-(f).

ties between the spectra of coronene and circumcoronene, and their differences with the spectra of HBC (smaller energy difference between lowest two-photon and onephoton states, much larger energy difference between the two-photon states) arise from the different characters of the edges, namely, zigzag versus armchair. [50]

To summarize, the energy gaps to the lowest onephoton optically allowed states, observed experimentally, can always be reproduced within the Hückel model by choosing an appropriate one-electron hopping integral $t$ (usually larger than realistic). However, as seen in the "TPA Measurement" subsection below, the same procedure, when extended to two-photon states, not only fails to give correct fits to experimentally observed energies of two-photon states, but actually predicts incorrect excited state ordering.

Experimental Linear Absorption versus PPPMRSDCI Calculations. The linear absorption measurements were performed with a UV/visible spectrophotometer (Varian Inc.) in dilute $(\sim 0.1 \mathrm{mM})$ solutions of Coronene and HBC in dichloroethane. The coronene sample was obtained from Sigma-Aldrich Chemical Co. and HBC was prepared using the method outlined in ref 51. We observed that the main features of absorption spectra do not change for concentrations between 0.01 and $1 \mathrm{mM}$, indicating that aggregation effects, if any, are minimal. Figure $3 \mathrm{a}, \mathrm{b}$ present the experimental solution phase linear absorption spectra of coronene and HBC, superimposed on our calculated PPP-MRSDCI absorption spectra. All calculated PPP spectra, here and in the following, are with uniform $t=2.4 \mathrm{eV}$. We find excellent match between the strong experimental absorption band and the calculated absorption band corresponding to transitions to the degenerate $1^{1} \mathrm{~B}_{2 u}^{+}$and $1^{1} \mathrm{~B}_{3 u}^{+}$states in both coronene and HBC. The weak splitting in the strong experimental absorption band, seen in both cases, confirms the existence of two underlying transitions that have lost their perfect degeneracy in the real systems. The much weaker absorptions (at $\sim 3.55 \mathrm{eV}$ in coronene and $\sim 3.2 \mathrm{eV}$ in $\mathrm{HBC}$ ), missing in the calculated absorption spectra, are to the "forbidden" $1^{1} \mathrm{~B}_{2 u}^{-}$and $1^{1} \mathrm{~B}_{3 u}^{-}$ states. Loss of degeneracy of the allowed transitions, and nonzero oscillator strengths of the "forbidden" transitions can be due to the couplings of electrons with vibronic modes as well as weak second neighbor electron hopping that are absent in our Hamiltonian. Neither of these interactions affect the relative ordering of the oneand two-photon states.

TPA Measurement. We conducted experimental measurements of TPA in $\lesssim 1 \mathrm{mM}$ solutions of coronene and HBC using dichloroethane as a solvent. High-intensity, 100 fs laser pulses with photon energies between 1.5-3 $\mathrm{eV}$ were obtained from titanium-sapphire pumped optical parametric amplifier (OPA) and used to measure the TPAs to states at energies 3.0-6.0 eV. The coronene and HBC solutions in $1 \mathrm{~mm}$ UV-quartz cuvettes were used for open-aperture "z-scan" measurement that allows extraction of TPA coefficient. [52] We employed a modified differential z-scan approach similar to the one discussed in ref 53 to enhance the sensitivity of our TPA measure- 


\begin{tabular}{l|cccc} 
molecule & $\mathrm{S}_{2}(\mathrm{eV})$ & $2^{1} \mathrm{~A}_{g}^{-}(\mathrm{eV})$ & $\mathrm{T}_{1}(\mathrm{eV})$ & $\xi_{1 \mathrm{e}-1 \mathrm{~h}}$ \\
\hline octatetraene & $4.50\left(4.40^{b}\right)$ & $3.42\left(3.59^{b}\right)$ & $1.65\left(1.73^{c}\right)$ & 0.33 \\
decapentaene & $4.13\left(4.02^{b}\right)$ & $3.06\left(3.10^{b}\right)$ & 1.52 & 0.30 \\
coronene & 4.14 & 3.96 & $2.38\left([2.37-2.40]^{d}\right)$ & 0.63 \\
HBC & 3.37 & 3.30 & 2.07 & 0.71 \\
circumcoronene & 2.67 & 2.75 & 1.50 & 0.60
\end{tabular}

TABLE II: The Calculated Energies of the One-Photon Optical State $\mathrm{S}_{2}$, the Lowest Two-Photon State $2^{1} \mathrm{~A}_{g}^{-}$, and the Lowest Triplet State $\mathrm{T}_{1}$ for Linear Polyenes and the Polycyclic Molecules of Fig. $1^{p}$

${ }^{a}$ The numbers in parentheses are experimental quantities. The last column gives the fractional contribution by $1 \mathrm{e}-1 \mathrm{~h}$ HF excitations to the $2^{1} \mathrm{~A}_{g}^{-} .{ }^{b}$ Table II, ref $1{ }^{c}$ Reference $48,{ }^{d}$ Reference 49.

ments. The z-scan measurements with the solvent alone were used as the "control" for the data reported here.

In Figure 3. we show the experimental TPA spectrum of coronene, where we have included the linear absorption for comparison. The TPA spectrum of Figure 3 ; is different from the Hückel TPA spectrum for coronene in Figure $2 \mathrm{~d}$ in three distinct ways: (i) resonances to twophoton states below the allowed one-photon states are observed, (ii) the experimental spectrum shows many more structures, and (iii) the intensities of the TPAs are higher at higher energy. The TPA spectrum of HBC, shown in Figure $3 \mathrm{~d}$ is very similar to that of coronene, with once again nonzero TPA below the strong one-photon allowed linear absorption. The strengths of the TPAs, both below the optical gap and at higher energies, are significantly higher for $\mathrm{HBC}$ with larger molecular size. A similar increase in the intensities of the TPAs for small chain lengths is also observed in polyenes. [47] We again note the apparent high density of two-photon states in the energy region $4.0-5.0 \mathrm{eV}$, in contradiction to the prediction of the Hückel theory (see Figure 2e). The energydependence of the intensities for the TPAs in HBC is also similar to that in coronene.

PPP-MRSDCI Calculations of TPA. Figure 3e,f presents the calculated TPA spectra (eq 3 . Dipole matrix elements were evaluated using the PPP-MRSDCI many-body wave functions. The calculated linear spectra have been included in the figures for comparison. The $\chi_{\text {avg }}^{(3)}(T P A)$ spectrum has resonances to both ${ }^{1} \mathrm{~A}_{g}^{-}$and ${ }^{1} \mathrm{~B}_{1 g}^{-}$states (see arrows in Figure 3 e,f).

In agreement with experiments, the calculated lowest two-photon state, the $2^{1} \mathrm{~A}_{g}^{-}$, occurs below the optical $1^{1} \mathrm{~B}_{2 u}^{+}$and $1^{1} \mathrm{~B}_{3 u}^{+}$states in coronene (by $0.18 \mathrm{eV}$ ) as well as in $\mathrm{HBC}$ (by $0.08 \mathrm{eV}$ ). Comparing Figure 3d,f we find excellent matches between the energy locations of the experimental and calculated two-photon resonances in HBC. The fit is less impressive for coronene. The high densities of two-photon states observed in both coronene and $\mathrm{HBC}$ agree with the occurrence of many close lying ${ }^{1} \mathrm{~A}_{g}^{-}$and ${ }^{1} \mathrm{~B}_{1 g}^{-}$states in the same energy range within the PPP model. The calculations also reproduce the intensity profile of the experimental TPAs, with the intensity at higher energies larger than that at lower energies.

The calculated lowest two-photon state is slightly above the lowest one-photon state in circumcoronene (see Table II below), with, however, an energy separation $(0.08 \mathrm{eV})$ much smaller than expected within oneelectron theory. The almost "normal" excited state ordering in circumcoronene is likely due to a weak breakdown of the MRSDCI approximation, which becomes less appropriate with increasing size.[16] With 54 C-atoms, circumcoronene is more than twice the size of coronene and is near the memory limit of MRSDCI, viz., 60 atoms.

\section{— DISCUSSION AND CONCLUSIONS}

Correlation effects in 2D, though substantial, are weaker than in linear chains. TableIs culated PPP energies of the lowest one- and two-photon spin singlet states, the lowest triplet state (hereafter $\mathrm{T}_{1}$ ), and the wave function characteristics of the lowest two-photon state for 1,3,5,7-octatetraene, 1,3,5,7,9decapentaene, and the three molecules we have investigated here. The polyene full CI calculations were done with hopping integrals $t=2.6$ and $2.2 \mathrm{eV}$ for the double and single bonds, respectively. 25] We have also included in Table II all experimental energy values that were available to us from literature. We note the excellent agreements between all theoretical and experimental 

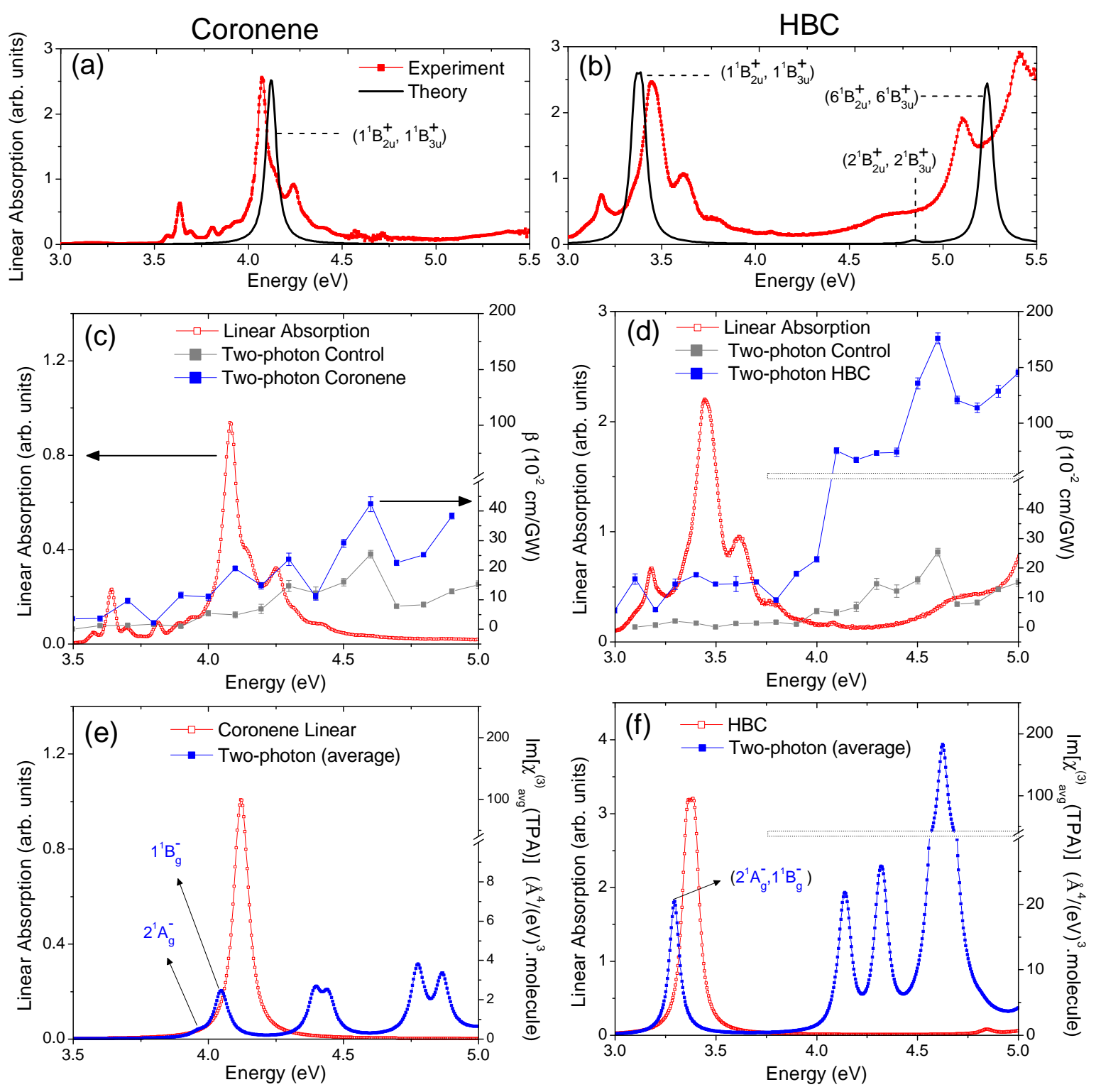

FIG. 3: (a) Experimental (red) and calculated (black) optical absorption spectra of coronene; calculations are within the PPP model. The weak absorption at $\sim 3.5 \mathrm{eV}$, missed in the calculation, is to a state that is optically dark within the purely electronic PPP Hamiltonian with nearest-neighbor electron hopping. (b) Same as in (a) for HBC. The lowest energy weak absorption is again to a dark state. (c) Experimental linear (red) and TPA (blue) spectra of coronene. The gray curve gives the TPA due to the solvent. Notice the significant TPA of coronene below the linear absorption edge. (d) Same as (c) for HBC. (e) Calculated linear absorption (red) and average TPA $\operatorname{Im}\left[\chi_{\text {avg }}^{(3)}(T P A)\right]$ (blue) for coronene. Two-photon resonances are to both ${ }^{1} \mathrm{~A}_{g}$ and ${ }^{1} \mathrm{~B}_{1 g}$ states (arrows) which are nondegenerate within the PPP model. (f) Same as in (e), for HBC. $\delta$ in eq 2 is $0.03 \mathrm{eV}$ for the TPA spectra of panels (e) and (f).

energies for the two polyenes. Equally remarkable agreement is found between the calculated and experimental triplet energies of coronene. The $2^{1} \mathrm{~A}_{q}^{-}$in the polyenes is known to be a quantum-entangled "two-triplet" state [4, 5] whose energy is nearly twice that of $\mathrm{T}_{1}$. By contrast, $2^{1} \mathrm{~A}_{g}^{-}$in the PAH molecules in all cases is less than twice the energy of their $T_{1}$. The low energy of the $2^{1} \mathrm{~A}_{g}^{-}$in polyenes, relative to the $1^{1} \mathrm{~B}_{u}^{+}$, is best understood within valence bond (VB) theory. [4, 5. For strong Hubbard $U$, the ground state within VB theory is predominantly covalent, i.e., its wave function is dominated by VB diagrams in which all carbon atom $p_{z^{-}}$ orbitals are singly occupied. Optical absorption within tight-binding Hamiltonians necessarily requires chargetransfer between carbon atoms, 4, 5, which then implies that the $1^{1} \mathrm{~B}_{u}^{+}$is dominated by ionic VB diagrams with at least one pair of $\mathrm{C}^{+} \mathrm{C}^{-}$ion pair. Two-photon states are reached by a second charge-transfer excitation from 
a virtual ionic state, which can create yet another $\mathrm{C}^{+} \mathrm{C}^{-}$ ion pair, or annihilate the original ion pair, creating an excited state that is also covalent, but orthogonal to the ground state. 4, 5] For nonzero Hubbard $U$, the lowest ionic states are necessarily higher in energy than the lowest covalent states. There is a one-to-one correspondence between this qualitative VB picture and the CI approach within the MO basis, in that the $1^{1} \mathrm{~B}_{u}^{+}$, obtained by a single charge-transfer from the ground state in configuration space, is an $1 \mathrm{e}-1 \mathrm{~h}$ excitation, while the $2^{1} \mathrm{~A}_{g}^{-}$has strong contributions from $2 \mathrm{e}-2 \mathrm{~h}$ excitations. [1, 3 6, 10, 17, 19, 20. Since 1e-1h excitations, however, dominate the $2^{1} \mathrm{~A}_{g}^{-}$wave function in the $2 \mathrm{D}$ compounds, especially in $\mathrm{HBC}$, the analogy with the above VB qualitative picture suggests that the $2^{1} \mathrm{~A}_{g}^{-}$in the $\mathrm{PAH}$ is presumably more ionic than in 1D. While investigation of the ionicity of excited states of such large molecules is a formidable problem, we note that within VB theory large covalent character (small ionicity) of the $2^{1} \mathrm{~A}_{g}^{-}$necessarily requires large covalent character of the ground state, which can be investigated even for large molecules within the PPP model using a variety of approximate methods such as quantum Monte Carlo. We intend to investigate the relative covalent characters of polyenes and the present $\mathrm{PAH}$ molecules in the future using one such approximate method, the Path Integral Renormalization Group, used previously by one of us in a different context. 54

To conclude, we have implemented very high level correlated-electron calculations for the one- and twophoton energy states and absorption spectra of three PAH molecules: coronene, HBC and circumcoronene, all possessing $D_{6 h}$ point group symmetry. For coronene and HBC we have also performed precise one- and twophoton optical absorption experiments. The calculated one-photon energies of the $1^{1} \mathrm{~B}_{2 u}^{+}$and $1^{1} \mathrm{~B}_{3 u}^{+}$give excellent fits to the experimental transition energies of these states in both coronene and HBC. For the first time, two-photon states below the lowest optically allowed onephoton state are demonstrated in 2D PAHs, in complete contradiction to the predictions of the one-electron Hückel model. Theoretical determination of subgap twophoton states can be achieved only by taking into account electron correlation effects that are an order higher than the 1e-1h excitonic interactions. 1, 3, 6, 10,17, 19, 20, Our theoretical and experimental works, taken together, indicate that while the consequences of $\mathrm{e}-\mathrm{e}$ interactions in the PAHs are weaker than in polyenes, these effects are nevertheless significant. In particular, correlation effects in PAHs appear to be stronger than in the phenyl group-containing $\pi$-conjugated quasi-1D polymers poly(paraphenylene) and poly-(paraphenylenevinylene), in which the $2^{1} \mathrm{~A}_{g}^{-}$occurs above the $1^{1} \mathrm{~B}_{u}^{+}$state. [55]

In the future we are planning to perform similar theoretical as well as experimental investigations of $\mathrm{PAH}$ molecules larger than the ones considered here. Such analyses can potentially open up an effective means to determine the effective short-range Hubbard $U$ in graphene,
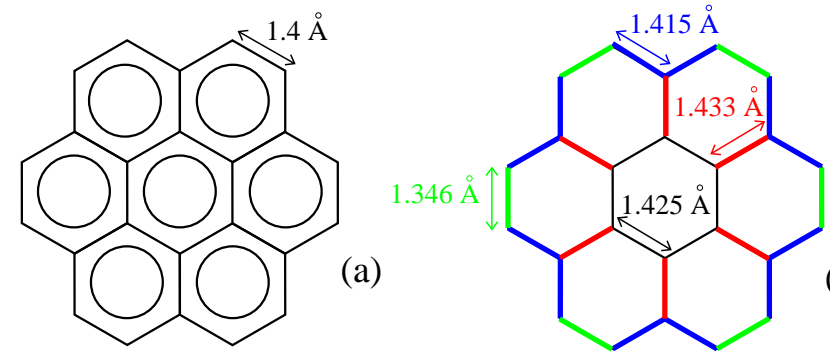

(b)

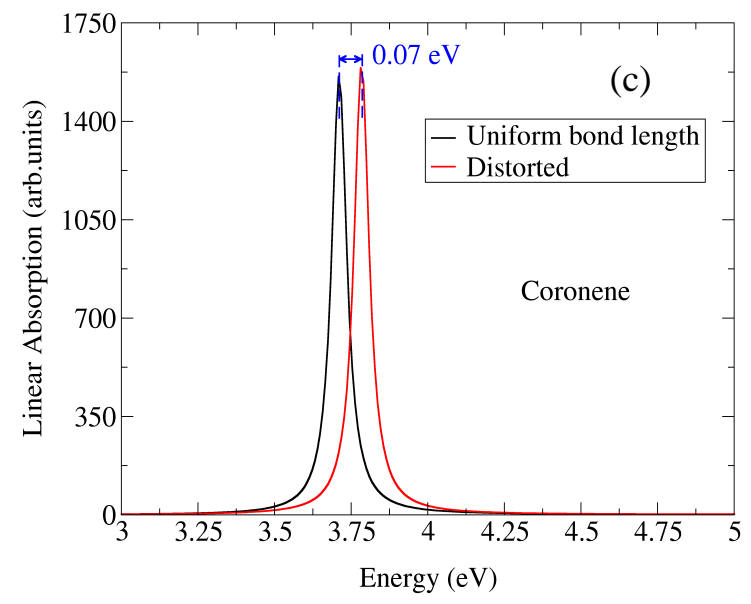

FIG. 4: (a) Coronene molecule with uniform bond lengths of $1.4 \AA$ assigned to all $\mathrm{C}-\mathrm{C}$ bonds. (b) Actual bond lengths of coronene, from ref 56. Note that $D_{6 h}$ symmetry is maintained. (c) Ground state linear absorption corresponding to (a) and (b), performed using the single-CI approximation.

which as we pointed out in the Introduction, remains controversial.

\section{- APPENDIX}

\section{Consequences of Deviations from Idealized Geometry: The Case of Coronene}

In our theoretical analyses of e-e correlation effects, we have used idealized geometries with uniform $\mathrm{C}-\mathrm{C}$ bond lengths of $1.4 \AA$ and hopping integrals $t=2.4 \mathrm{eV}$. In reality, the $\mathrm{C}-\mathrm{C}$ bonds deviate from this idealized length, and these deviations can in principle influence our overall results. Deviations from the idealized geometry are, however, expected to be small for the aromatic molecules in question. The experimental bond lengths are available only for coronene. Here we demonstrate explicitly for this case that the expected changes in energies due to geometry modifications are negligible, and in no way affects our main conclusion.

In Figure 4a,b we have shown the coronene molecule with its idealized uniform bond lengths, and with the actual bond lengths, 56 respectively. We calculated all hopping integrals from the known bond length-hopping integral relationship, $t_{C-C}=-2.4+3.20\left(R_{C-C}-1.4\right)$, 
57. where $R_{C-C}$ is the $\mathrm{C}-\mathrm{C}$ bond length in $\AA$. The calculated hopping integrals in descending order are 2.57, $2.35,2.32$, and $2.29 \mathrm{eV}$, respectively. The $V_{i j}$ were calculated as defined in eq11 within the text. Figure 4 c shows the ground state linear absorption within the single-CI for the geometries of Figure $4 \mathrm{a}, \mathrm{b}$. Deviation from equal bond lengths blue shifts the main absorption band very weakly (the energy of the optical state is slightly smaller than what is obtained within the MRSDCI, but this is of no concern here).

As mentioned above, experimental bond lengths are currently not available for HBC. Based on our results for coronene, we believe, however, that it is safe to assume that any deviations in energies due to weak distortions from idealized geometries will not affect our overall qualitative conclusion about the reversed excited state ordering, which cannot be obtained without explicit inclusion of strong $\mathrm{e}-\mathrm{e}$ interactions, and which is the core theme of our work.

\section{AUTHOR INFORMATION}

\section{Corresponding Author}

*E-mail: karana@physics.arizona.edu; Phone: +1-520621-6798; Fax: +1-520-621-4721.

Notes

The authors declare no competing financial interest.

\section{ACKNOWLEDGMENTS}

This work was supported by NSF Grant No. CHE1151475. A.R. acknowledges the support from the DoD SMART program. A.Sh. and S.M. acknowledge support from the Indo-US Science and Technology Forum Award 37-2012/2013-14.
[1] Hudson, B. S.; Kohler, B. E.; Schulten, K. Linear Polyene Electronic-Structure and Potential. Excited States. 1982, $6,1-95$.

[2] Christensen, R. L.; Galinato, M. G. I.; Chu, E. F.; Howard, J. N.; Broene, R. D.; Frank, H. A. Energies of Low-Lying Excited States of Linear Polyenes. J. Phys. Chem. A 2008, 112, 12629-12636.

[3] Schulten, K.; Karplus, M. On the Origin of a Low-Lying Forbidden Transition in Polyenes and Related Molecules. Chem. Phys. Lett. 1972, 14, 305-309.

[4] Ramasesha, S.; Soos, Z. G. Correlated States in Linear Polyenes, Radicals, and Ions: Exact PPP Transition Moments and Spin Densities. J. Chem. Phys. 1984, 80, 3278-3287.

[5] Tavan, P.; Schulten, K. Electronic Excitations in Finite and Infinite Polyenes. Phys. Rev. B 1987, 36, 4337-4358.

[6] Baeriswyl, D.; Campbell, D. K.; Mazumdar, S. Conjugated Conducting Polymers; Kiess, H., Ed.; Springer: Berlin, 1992.

[7] Barcza, G.; Barford, W.; Gebhard, F.; Legeza, O. Excited States in Polydiacetylene Matrix Renormalization Group Study. Phys. Rev. B 2013, 87, 245116-245131.

[8] Pariser, R.; Parr, R. G. A Semi-Empirical Theory of the Electronic Spectra and Electronic Structure of Complex Unsaturated Molecules. II. J. Chem. Phys. 1953, 21, $767-776$.

[9] Pople, J. A. Electron Interaction in Unsaturated Hydrocarbons. Trans. Faraday Soc. 1953, 49, 1375-1385.

[10] Schreiber, M.; Silva-Junior, M. R.; Sauer, S. P. A.; Thiel, W. Benchmarks for Electronically Excited States: CASPT2, CC2, CCSD, and CC3. J. Chem. Phys. 2008, 128, 134110-134134.

[11] Silva-Junior, M. R.; Schreiber, M.; Sauer, S. P. A.; Thiel, W. Benchmarks for Electronically Excited States: Basis Set Effects on CASPT2 Results. J. Chem. Phys. 2010, 133, 174318-174330.

[12] Starcke, J. H.; Wormit, M.; Schirmer, J.; Dreuw, A. How Much Double Excitation Character Do the Lowest
Excited States of Linear Polyenes Have? Chem. Phys. 2006, 329, 39-49.

[13] Knippenberg, S.; Rehn, D. R.; Wormit, M.; Starcke, J. H.; Rusakova, I. L.; Trofimov, A. B.; Dreuw, A. Calculations of Nonlinear Response Properties Using the Intermediate State Representation and the AlgebraicDiagrammatic Construction Polarization Propagator Approach: Two-Photon Absorption Spectra. J. Chem. Phys. 2012, 136, 064107-064121.

[14] Krauter, C. M.; Pernpointner, M.; Dreuw, A. Application of the Scaled-Opposite-Spin Approximation to the Algebraic Diagrammatic Construction Schemes of Second Order. J. Chem. Phys. 2013, 138, 044107-044118.

[15] Silva-Junior, M. R.; Schreiber, M.; Sauer, S. P. A.; Thiel, W. Benchmarks for Electronically Excited States: TimeDependent Density Functional Theory and Density Functional Theory Based Multireference Configuration Interaction. J. Chem. Phys. 2008, 129, 104103-104116.

[16] Schmidt, M.; Tavan, P. Electronic Excitations in Long Polyenes Revisited. J. Chem. Phys. 2012, 136, 124309-124321.

[17] Tavan, P.; Schulten, K. Correlation Effects in the Spectra of Polyacenes. J. Chem. Phys. 1979, 70, 5415-5421.

[18] Raghu, C.; Anusooya Pati, Y.; Ramasesha, S. DensityMatrix Renormalization-Group Study of Low-Lying Excitations of Polyacene within a Pariser-Parr-Pople Model. Phys. Rev. B 2002, 66, 035116-035126.

[19] Marian, C. M.; Gilka, N. Performance of the Density Functional Theory/Multireference Configuration Interaction Method on Electronic Excitation of Extended $\pi$-Systems. J. Chem. Theory. Comput. 2008, 4, $1501-1515$.

[20] Knippenberg, S.; Starcke, J. H.; Wormit M.; Dreuw, A. The Low-Lying Excited States of Neutral Polyacenes and Their Radical Cations: A Quantum Chemical Study Employing the Algebraic Diagrammatic Construction Scheme of Second Order. Mol. Phys. 2010, 108, 2801-2813.

[21] Geim, A. K.; Novoselov, S. The Rise of Graphene. Nat. 
Mat. 2007, 6, 183-191.

[22] Castro Neto, A. H.; Guinea, F.; Peres, N. M. R.; Novoselov, K. S.; Geim, A. K. The Electronic Properties of Graphene. Rev. Mod. Phys. 2009, 81, 109-162.

[23] Elias, D. C.; Gorbachev, R. V.; Mayorov, A. S.; Morozov, S. V.; Zhukov, A. A.; Blake, P.; Ponomarenko, L. A.; Grigorieva, I. V.; Novoselov, K. S.; Guinea, F.; et al. Dirac Cones Reshaped by Interaction Effects in Suspended Graphene. Nat. Phys. 2011, 7, 701-704.

[24] Kotov, V. N.; Uchoa, B.; Pereira, V. M.; Guinea, F.; Castro Neto, A. H. Electron-Electron Interactions in Graphene: Current Status and Perspectives. Rev. Mod. Phys. 2012, 84, 1067-1125.

[25] Chandross, M.; Mazumdar, S. Coulomb Interactions and Linear, Nonlinear, and Triplet Absorption in Poly(paraphenylenevinylene). Phys. Rev. B 1997, 55, 1497-1504.

[26] Sony, P.; Shukla, A. Large-Scale Correlated Calculations of Linear Optical Absorption and Low-Lying Excited States of Polyacenes: Pariser-Parr-Pople Hamiltonian. Phys. Rev. B 2007, 75, 155208-155229.

[27] Wang, Z. D.; Zhao, H. B.; Mazumdar, S. Quantitative Calculations of the Excitonic Energy Spectra of Semiconducting Single-Walled Carbon Nanotubes within a $\pi$ Electron Model. Phys. Rev. B 2006, 74, 195406-195411.

[28] Yang, L.; Deslippe, J.; Park, C.-H.; Cohen, M. L.; Louie, S. G. Excitonic Effects on the Optical Response of Graphene and Bilayer Graphene. Phys. Rev. Lett. 2009, 103, 186802-186805.

[29] Spataru, C. D.; Ismail-Beigi, S.; Benedict, L. X.; Louie, S. G. Excitonic Effects and Optical Spectra of SingleWalled Carbon Nanotubes. Phys. Rev. Lett. 2004, 92, 077402-077405.

[30] Chang, E.; Bussi, G.; Ruini, A.; Molinari, E. Excitons in Carbon Nanotubes: An Ab Initio Symmetry-Based Approach. Phys. Rev. Lett. 2004, 92, 196401-196404.

[31] Prezzi, D.; Varsano, D.; Ruini, A.; Marini, A.; Molinari, E. Optical Properties of Graphene Nanoribbons: The Role of Many-Body Effects. Phys. Rev. B 2008, 77, 041404-041407.

[32] Rohlfing, M.; Louie S. G. Optical Excitations in Conjugated Polymers. Phys. Rev. Lett. 1999, 82, 1959-1962.

[33] Ramasesha, S.; Pati, S. K.; Shuai, Z.; Brédas, J. L. The Density Matrix Renormalization Group Method: Application to the Low-Lying Electronic States in Conjugated Polymers. Adv. Quantum Chem. 2000, 38, 121-215.

[34] Bursill, R. J.; Barford, W. Large-Scale Numerical Investigation of Excited States in Poly(paraphenylene). Phys. Rev. B 2002, 66, 205112-205119.

[35] Buenker, R. J.; Peyerimhoff, S. D. Individualized Configuration Selection in CI Calculations with Subsequent Energy Extrapolation. Theor. Chim. Acta. 1974, 35, $33-58$.

[36] Tavan, P.; Schulten, K. The Low-Lying Electronic Excitations in Long Polyenes: A PPP-MRD-CI Study. J. Chem. Phys. 1986, 85, 6602-6609.

[37] Ghosh, H.; Shukla, A.; Mazumdar, S. ElectronCorrelation-Induced Transverse Delocalization and Longitudinal Confinement in Excited States of PhenylSubstituted Polyacetylenes. Phys. Rev. B 2000, 62, 12763-12774.

[38] Shukla, A. Correlated Theory of Triplet Photoinduced Absorption in Phenylene-Vinylene Chains. Phys. Rev. B 2002, 65, 125204-125209.
[39] Shukla, A.; Ghosh, H.; Mazumdar, S. Theory of ExcitedState Absorption in Phenylene-Based $\pi$-Conjugated Polymers. Phys. Rev. B 2003, 67, 245203-245211.

[40] Shukla, A. Theory of Two-Photon Absorption in Poly(diphenyl) Polyacetylenes. Chem. Phys. 2004, 300, 177-188.

[41] Shukla, A. Theory of Nonlinear Optical Properties of Phenyl-Substituted Polyacetylenes. Phys. Rev. B 2004, 69, 165218-165227.

[42] Sony, P.; Shukla, A. Photoinduced Absorption in Disubstituted Polyacetylenes: Comparison of Theory with Experiments. Phys. Rev. B 2005, 71, 165204-165208.

[43] Aryanpour, K.; Sheng, C. X.; Olejnik, E.; Pandit, B.; Psiachos, D.; Mazumdar, S.; Vardeny, Z. V. Evidence for Excimer Photoexcitations in an Ordered $\pi$-Conjugated Polymer Film. Phys. Rev. B 2011, 83, 155124-155128.

[44] Boyd, R. W. Nonlinear Optics; Academic Press, Inc.: San Diego, CA, 1992.

[45] Heflin, J. R.; Wong, K. Y.; Zamani-Khamiri, O.; Garito, A. F. Nonlinear Optical Properties of Linear Chains and Electron-Correlation Effects. Phys. Rev. B 1988, 38, 1573-1576.

[46] McWilliams, P. C. M.; Hayden, G. W.; Soos, Z. G. Theory of Even-Parity States and Two-Photon Spectra of Conjugated Polymers. Phys. Rev. B 1991, 43, 9777-9791.

[47] Mazumdar, S.; Guo, F. Intensities of Two-Photon Absorptions to Low-Lying Even-Parity States in LinearChain Conjugated Polymers. Phys. Rev. B 1994, 49, 10102-10112.

[48] Allan, M.; Neuhaus L.; Haselbach E. (all-E)-1,3,5,7Octatetraene: Electron-Energy-Loss and ElectronTransmission Spectra. Helv. Chim. Acta 1984, 67, 1776-1782.

[49] Abouaf, R.; Díaz-Tendero S. Electron Energy Loss Spectroscopy and Anion Formation in Gas Phase Coronene. Phys. Chem. Chem. Phys. 2009, 11, 5686-5694.

[50] Güçlü, A. D.; Potasz, P.; Hawrylak, P. Excitonic Absorption in Gate-Controlled Graphene Quantum Dots. Phys. Rev. B 2010, 82, 155445-155449.

[51] Rathore, R.; Burns, C. L. A Practical One-Pot Synthesis of Soluble Hexa-Peri-Hexabenzocoronene and Isolation of Its Cation-Radical Salt. J. Org. Chem. 2003, 68, 4071-4074.

[52] Sheik-Bahae, M.; Said, A. A.; Wei, T.-H.; Hagan, D. J.; Van Stryland, E. W. Sensitive Measurement of Optical Nonlinearities Using a Single Beam. IEEE. J. Quantum Electron. 1990, 26, 760-769.

[53] Ménard, J. M.; Betz, M.; Sigal, I.; van Driel, H. M. Single-Beam Differential Z-Scan Technique. Appl. Opt. 2007, 46, 2119-2122.

[54] Dayal, S.; Clay, R. T.; Mazumdar, S. Absence of LongRange Superconducting Correlations in the Frustrated Half-Filled-Band Hubbard Model. Phys. Rev. B 2012, 85, 165141-165148.

[55] Soos, Z. G.; Ramasesha, S.; Galvão, D. S. Band to Correlated Crossover in Alternating Hubbard and PariserParr-Pople Chains: Nature of the Lowest Singlet Excitation of Conjugated Polymers. Phys. Rev. Lett. 1993, 71, 1609-1612.

[56] Fawcett, J. K. The determination and refinement of the molecular structures of some organic compounds; http://hdl.handle.net/2429/38382, 1965.

[57] Ducasse, L. R.; Miller, T. E.; Soos, Z. G. Correlated 
States in Finite Polyenes: Exact PPP Results. J. Chem. Phys. 1982, 76, 4094-4104. 